\title{
Criminologie
}

\section{Autocorrélation spatiale et déplacement de la criminalité}

\section{Daniel Élie et Pierre Legendre}

Volume 25, numéro 2, 1992

Nouvelles connaissances et nouvelles questions en criminologie

URI : https://id.erudit.org/iderudit/017326ar

DOI : https://doi.org/10.7202/017326ar

Aller au sommaire du numéro

Éditeur(s)

Les Presses de l'Université de Montréal

ISSN

0316-0041 (imprimé)

1492-1367 (numérique)

Découvrir la revue

Citer cet article

Élie, D. \& Legendre, P. (1992). Autocorrélation spatiale et déplacement de la criminalité. Criminologie, 25(2), 139-154. https://doi.org/10.7202/017326ar

\section{Résumé de l'article}

This research examines spatial autocorrelation and crime displacement as facts and concepts. The objective of the study concerns both the methodological theory and the practice of applied research in criminology. The two expressions used in the title can be considered a fragmentation of the concept of turbulence. The authors examine the influence of the presence of spatial autocorrelation in the data on tests of the significance of statistics. They attempt to detect it in a part of central Montreal by examining the distribution and evolution of the two types of crime: armed robbery and burglary. Three methods are used: the known coefficients and correlograms, spatial grouping with forces contiguity, and trend surface analysis. The last two methods lead to convincing results. On the other hand, the displacement could not be observed during the time studied. In conclusion, they consider the implications for the methodological practices and usual research strategies in criminology. 


\section{AUTOCORRÉLATION SPATIALE ET DÉPLACEMENT DE LA CRIMINALITÉ Daniel Élie ${ }^{1}$ \\ Pierre Legendre ${ }^{2}$}

This research examines spatial autocorrelation and crime displacement as facts and concepts. The objective of the study concerns both the methodological theory and the practice of applied research in criminology. The two expressions used in the title can be considered a fragmentation of the concept of turbulence. The authors examine the influence of the presence of spatial autocorrelation in the data on tests of the significance of statistics. They attempt to detect it in a part of central Montreal by examining the distribution and evolution of the two types of crime: armed robbery and burglary. Three methods are used: the known coefficients and correlograms, spatial grouping with forces contiguity, and trend surface ana. lysis. The last two methods lead to convincing results. On the other hand, the displacement could not be observed during the time studied. In conclusion, they consider the implications for the methodological practices and usual research strategies in criminology.

\section{REMERCIEMENTS}

Les auteurs remercient leur assistante de recherche, Mme Manon Lortie, pour son travail remarquable. $M$. Théophile Niyonsenga, chercheur postdoctoral attaché au laboratoire de $P$. Legendre, a produit les cartes interpolées. M. Alain Vaudor, informaticien attaché au laboratoire de $P$. Legendre et auteur des programmes « Autocorrelation » (analyse des corrélogrammes spatiaux) et «Biogéo» (groupement sous contrainte de contiguîté spatiale) utilisés dans cette recherche, a largement contribué à l'analyse de nos données. M. Vaudor est décédé le 31 octobre 1991 à l'âge de 46 ans. Le progiciel d'analyse multidimensionnelle et spatiale qu'il a produit au cours de sa carrière d'informaticien est disponible chez P. Legendre.

1. Professeur, École de criminologie, Université de Montréal, case postale 6128 , succursale A, Montréal, Québec, H3C 3J7, Canada.

2. Professeur titulaire, Département de sciences biologiques, Université de Montréal, case postale 6128, succursale A, Montréal, Québec, H3C 357, Canada. 


\section{INTRODUCTION}

Les études consacrées à l'analyse spatiale ou à l'écologie sociale ont connu un sort très variable en criminologie. Ainsi, il est indéniable que les travaux de l'École de Chicago autour de Shaw et McKay (1931) ont laissé des traces profondes et ont contribué, au moins, à articuler les débats de base en sociologie de la criminalité. Les réactions à ce courant au cours des années ont été assez vives et ont entraîné un certain désengagement de l'ensemble des chercheurs. Pourtant, les relations statistiques entre les taux de crime dans un territoire et les caractéristiques socio-démographiques et économiques demeurent les données autour desquelles s'est conçue Ia pensée positiviste en criminologie depuis la fin du XIX $X^{e}$ siècle. On constate toutefois dans le domaine de l'écologie du crime des résurgences particulièrement remarquables. Il suffit de citer, à titre d'exemple, les travaux de Brantingham et Brantingham (1981).

Il faut cependant admettre que, dans ce domaine, les résultats ont été jusqu'à présent relativement minces et plutôt épars: des corrélations difficilement interprétables, des relations ou des constats peu généralisables. On relève également que la prise en charge de manière officielle d'une information aussi importante que l'autocorrélation spatiale est peu présente, à notre connaissance, dans les travaux visibles et récents. Nous nous sommes proposés, dans une recherche exploratoire, de faire avancer cet aspect de la question en examinant les possibilités offertes par ce type d'analyse. Il s'agit donc de considérer l'approche écologique ou spatiale dans une optique renouvelée en espérant faire avancer méthode et théorie.

Après avoir énoncé le problème, nous décrivons le phénomène d'autocorrélation spatiale et ses implications; nous présentons ensuite la recherche réalisée, ses résultats, et nous en tentons une interprétation.

\section{$1^{\circ} \quad$ Le problème}

Nous avons voulu repérer et étudier le déplacement de la criminalité dans le centre-ville de Montréal. L'idée de base peut se résumer comme suit. La localisation géographique des crimes, leur environnement, procurent certainement beaucoup de renseignements, mais le déplacement de la criminalité représente une donnée encore peu exploitée. Cette réalité est incontournable si l'on veut ameliorer, par exemple, l'évaluation de l'effet des programmes de prévention; elle l'est encore si l'on entend raffiner la mise à l'épreuve de nombre d'hypothèses 
émises au sujet des liens supposés entre l'évolution des criminalités et certains faits sociaux et urbains. La question pourrait être: la criminalité répond-elle par ses déplacements aux mouvements des variables économiques ou sociales, ou possède-t-elle sa propre dynamique? La réponse à cette question dépendra éventuellement de l'échelle spatiale à laquelle l'étude est réalisée. Nous formulons donc dans cette prérecherche, et pour faire avancer le débat, une hypothèse très simple, à savoir: lorsqu'ils se produisent, les déplacements de certaines formes de criminalité ne sont pas aléatoires; ils répondent en partie à une loi d'autocorrélation spatiale et en partie à d'autres facteurs présents dans le nouveau micromilieu, facteurs qu'il reste à identifier. Nous croyons que ce nouveau type d'analyse du deplacement peut avoir une valeur heuristique et apporter un éclairage décisif sur les résultats de nombre de recherches antérieures. En effet, on peut penser que les corrélations entre l'évolution de toute forme de criminalité et les faits sociaux qui l'entourent deviendront beaucoup plus facilement interprétables dans cette situation d'observation. Ce dernier point sera plus facilement admis après que nous aurons défini, dans les paragraphes qui suivent, le phénomène d'autocorrélation et ses implications dans l'utilisation des méthodes statistiques.

\section{$2^{\circ}$ L'autocorrélation spatiale, ses implications et son utilisation}

Nous tenterons d'abord de montrer que les tests statistiques classiques ne sont pas toujours adéquats pour l'étude des phénomènes écologiques présentant une structure spatiale. Ceci justifiera l'utilisation de méthodes différentes lorsque l'intérêt de la recherche réside dans la nature même de la structure spatiale (c'est-à-dire, autocorrélation).

Dans les analyses d’inférence statistique classiques, l'un des postulats fondamentaux de l'épreuve d'hypothèse est l'indépendance des observations. L'existence même d'une structure spatiale dans le champ d'échantillonnage implique que cette condition fondamentale n'est pas remplie parce que tout phénomène écologique localisé en un point quelconque peut exercer une influence sur les autres points proches ou même à quelque distance.

Une variable est dite autocorrélée (ou régionalisée) lorsqu'il est possible de prédire les valeurs de cette variable en un point de l'espace (ou du temps) à partir des valeurs connues en d'autres points échantillonnés, dont les valeurs spatiales (ou temporelles) sont aussi connues. L'autocorrélation spatiale peut être décrite par une fonction mathématique dite de structure. 
L'autocorrélation n'est pas la même pour toutes les classes de distance entre les points d'échantillon; elle peut également être positive ou négative. Le plus souvent en écologie, elle est positive (ce qui signifie que la variable prend des valeurs similaires) pour les courtes distances entre les points. L'autocorrélation négative pour de courtes distances peut révéler un système d'évitement. Notons enfin que, si l'on ne trouve pas d'autocorrélation spatiale significative à une echelle spatiale donnée, ceci ne signifie pas qu'elle n'existe pas à une autre échelle.

La présence d'une autocorrélation positive à courte distance biaise des tests tels que les corrélations, la régression ou l'analyse de variance et rend les tests trop «libéraux» (Bivand, 1980; Cliff et Ord, 1981); ceci signifie qu'en présence d'autocorrélation positive les tests statistiques classiques aboutissent trop souvent à signaler des résultats significatifs alors qu'en fait ils peuvent ne pas l'être.

Lorsque la répartition géographique d'une variable (sa carte) suggère l'existence d'une structure spatiale, on peut être amené à vérifier statistiquement s'il existe une autocorrélation significative et à établir son type. Cette étude peut être entreprise pour deux raisons diamétralement opposées. D'une part, un chercheur peut vouloir prouver qu'il n'existe pas réellement d'autocorrélation afin de justifier l'emploi de techniques statistiques paramétriques. On peut également rechercher la preuve de l'existence d'une structure spatiale afin de mieux l'analyser. Notre démarche relève de cette seconde orientation.

\section{$3^{0}$ La recherche}

Pour les fins de la présente étude, nous avons choisi d'analyser la répartition et l'évolution spatiale des vols qualifiés et des cambriolages (introductions par effraction) au centre-ville de Montréal. Dans le décompte des vols qualifiés, nous avons retenu les catégories suivantes: vol au moyen d'une arme à feu; au moyen d'une arme offensive; autres vols qualifiés. Dans le décompte des cambriolages, les catégories comprennent les introductions par effraction dans les établissements commerciaux, dans les résidences et dans les autres types de locaux (par exemple les entrepôts). Ces types d'incidents ont été choisis sans intention théorique, mais plutôt pour des motifs de commodité et à cause du niveau de signalement qui nous a semblé suffisamment élevé pour traduire la tendance de fond et éviter les nombreux artefacts associés à ce type d'information statistique. 


\section{$4^{0} \quad$ Les données}

Les données de base proviennent de la police de la Communauté urbaine de Montréal (SPCUM). Il s'agit en fait de la liste complète de toutes les plaintes logées auprès de la police et des rapports d'événements d'un district administratif, avec la date et l'adresse exacte de l'incident. Le territoire choisi est celui du district de police $n^{\circ} 33$, qui représente une zone sensible du centre-ville de Montréal. La période d'observation s'étend du $1^{\mathrm{er}}$ janvier au 31 décembre de l'année 1989.

\section{$5^{\circ}$ Agencement du territoire}

Pour les fins de l'étude, le territoire a été subdivisé en 27 zones selon deux principaux critères. Nous avons tenu compte d'une part des frontières des secteurs de recensement afin de pouvoir éventuellement utiliser les informations s'y rapportant et déjà compilées par Statistique Canada. D'autre part, nous avons respecté autant que possible les frontières des zones "naturelles" afin d'obtenir autant que possible des résultats signifiants et interprétables en termes écologiques et spatiaux. C'est ainsi qu'on peut facilement identifier sur les cartes originelles la zone dite du "Village» (quadrilatère délimité par les rues SainteCatherine, Saint-Hubert, Sherbrooke et de la Visitation) ou celle du Vieux-Montréal, ou encore celle du «Quartier chinois ».

Par la suite, nous avons classifié chaque événement dans l'espace, c'est-à-dire dans l'une des 27 zones, au moyen de l'adresse; et dans le temps, pour les 22 périodes, au moyen de la date. L'intervalle de temps choisi est de 14 jours. En dénombrant les cas, on obtient ainsi deux matrices de fréquences, la première décrivant la situation des vols à main armée, et l'autre celle des cambriolages. En résumé, donc, nous avons constitué deux fichiers d'entrée, un pour chaque type de crime, comportant chacun 27 zones et 22 périodes; ces fichiers rassemblent, dans la forme requise pour le traitement des données, l'ensemble des informations contenues dans la liste initiale. Pour les fins de l'analyse spatiale, nous avons également constitué un fichier de $27 \times 2$ contenant les coordonnées géographiques des centroïdes approximatifs des 27 zones.

\section{$6^{0}$ Traitement des données}

Pour le traitement de ces données, les coefficients $I$ de Moran et c de Geary ont été utilisés pour évaluer l'autocorrélation spatiale. La présentation in extenso de ces coefficients entraînerait des considérations techniques assez détaillées et un exposé d'une ampleur qui 
dépasse le cadre de cet article. Nous soumettons donc leur interprétation usuelle et renvoyons le lecteur intéressé aux ouvrages de Cliff et Ord (1981) ou de Legendre et Legendre (1984a).

Le $I$ de Moran prend habituellement des valeurs comprises entre -1 (autocorrélation négative maximale : évitement extrême) et +1 (autocorrélation positive maximale: contagion maximale); dans certains cas, $I$ peut cependant excéder 1 . Son espérance, dans l'hypothèse d'une répartition spatiale complètement aléatoire, est $\mathrm{E}(1)=-(\mathrm{n}-1)^{-1}$ et non 0 comme dans le cas du coefficient de corrélation de Pearson.

Dans le cas du coefficient c de Geary, son espérance, dans l'hypothèse d'une répartition spatiale aléatoire, est $\mathrm{E}(\mathrm{c})=1$. Son échelle est cependant inversée par rapport au coefficient de Moran, puisque le $c$ de Geary est égal à 0 lorsque l'autocorrélation positive est maximale, alors qu'il peut atteindre une valeur positive quelconque, plus grande que 1 , si l'autocorrélation est négative.

Les corrélogrammes présentés sont des graphiques des valeurs d'autocorrélation (ordonnée) en fonction des différentes classes de distance (abscisse). Le corrélogramme est une bonne façon d'explorer le phénomène de répartition spatiale lorsqu'on n'a pas d'hypothèse $a$ priori à vérifier.

Un groupement avec contrainte a également été réalisé afin d'obtenir une partition des zones en groupes homogènes ainsi que les cartes des résultats que fournit le programme utilisé. Le programme Biogéo calcule un groupement agglomératif sous contrainte de contiguïté spatiale, tel qu'il est proposé par Legendre et Legendre (1984b), et présente les résultats sous forme d'une série de cartes, une pour chaque niveau de groupement. Puisque le groupement est fondé sur une matrice de similarités et que cette matrice est le plus souvent calculée à partir d'un grand nombre de variables, cette méthode peut donc être considérée comme une méthode de cartographie multidimensionnelle.

Le groupement agglomératif a été réalisế à l'aide d'un algorithme à liaison proportionnelle. La connexité est fixée par l'usager entre 0 (groupement à liens simples) et 1 (groupement à liens complets). Legendre (1987) a montré la stabilité des résultats du groupement obtenu avec contrainte à travers une large gamme de valeurs de connexité.

La composante spatiale a finalement été analysée au moyen d'une méthode classique en géographie, l'analyse des surfaces théoriques (trend surface analysis, en anglais). Cette méthode permet d'exprimer les variations d'une variable simple, en différents points de l'espace, 
comme une fonction des coordonnées spatiales des points d'échantillonnage:

$$
\hat{\mathrm{z}}=f(\mathrm{x}, \mathrm{y})
$$

où $\hat{z}$ représente les valeurs de la variable dépendante $z$ prédites par la fonction, alors que $\mathrm{x}$ et $\mathrm{y}$ représentent les valeurs des coordonnées spatiales des points d'échantillonnage dans un plan cartésien. Une fonction du premier degré $\hat{z}=b_{0}+b_{1} x+b_{2} y$, qui est un modèle linéaire, permet de faire passer un plan à travers les différentes valeurs de $\mathrm{z}$ sur la carte. Des fonctions d'un degré plus élevé telles que le pylonôme cubique

$\hat{z}=b_{0}+b_{1} x+b_{2} y+b_{3} x^{2}+b_{4} x y+b_{5} x^{2}+b_{6} x^{3}+b_{7} x^{2} y+b_{8} x y^{2}+b_{9} y^{3}$

permettent d'expliquer une plus grande fraction de la variation de $z$ dans les cas où la structure spatiale est plus complexe qu' un pian incliné. La méthode d'analyse des surfaces théoriques utilise la régression multiple comme instrument de calcul. On pourra consulter Ripley (1981) pour un exposé plus complet.

Le problème du choix du degré du polynôme et des termes qui doivent y être conservés est discuté par Legendre (1993). Lorsqu'on augmente l'ordre du polynôme, on pourrait par exemple tester si la fraction de variation expliqué par l'addition des nouveaux termes est significativement plus grande que zéro, comme en régression polynomiale. Pour la présente recherche, une procédure d'élimination descendante (backward elimination, en anglais) a été employée: à partir de l'équation cubique (2) ci-dessus, les termes les moins significatifs ont été éliminés par étapes successives, jusqu'à ce qu'il ne reste plus que des termes significatifs dans l'équation spatiale. Les variables $x$ et $y$ avaient été centrées avant le calcul des termes de degré supérieur $\left(x^{2}, x y\right.$, etc. $)$, de façon à éliminer une part non essentielle de la colinéarité entre les variables spatiales soumises à la procédure de régression.

Des cartes interpolées ont été produites, à l'aide du programme MacGRIDO $^{\text {TM }}$ (Rock Ware, Inc.), pour les valeurs prédites par les modèles spatiaux.

En résumé, l'analyse de la structure spatiale a été réalisée selon trois méthodes différentes, à savoir: par l'utilisation des coefficients d'autocorrélation spatiale et des corrélogrammes associés, par regroupement spatial et, enfin, par analyse des surfaces théoriques. 


\section{$7^{\circ} \quad$ Résultats et interprétation}

a) Première analyse: autocorrélation spatiale

L'examen de l'autocorrélation a été réalisé pour chaque période de deux semaines ainsi que pour l'ensemble des 11 mois, et ce, pour chacun des deux types de criminalité. Les résultats sont illustrés par les corrélogrammes présentés aux figures 1 et 2 à titre d'exemple.

Les structures spatiales les plus courantes sont le gradient, caractérisé par un corrélogramme de Moran comportant des valeurs positives

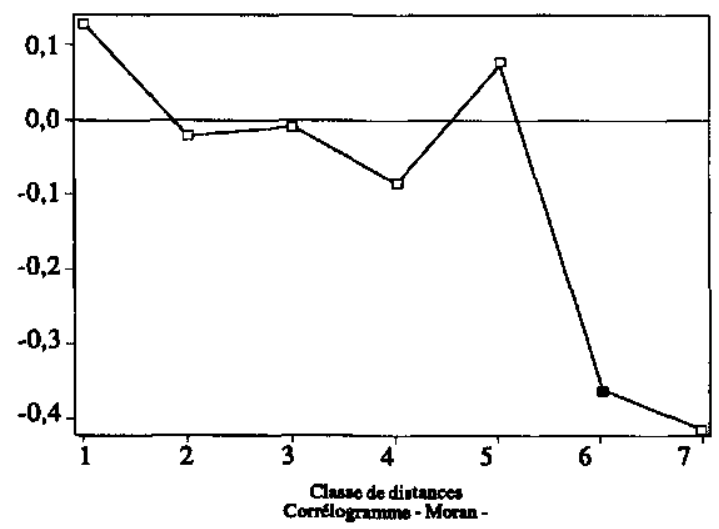

Figure 1 : Introductions par effraction, 11 mois

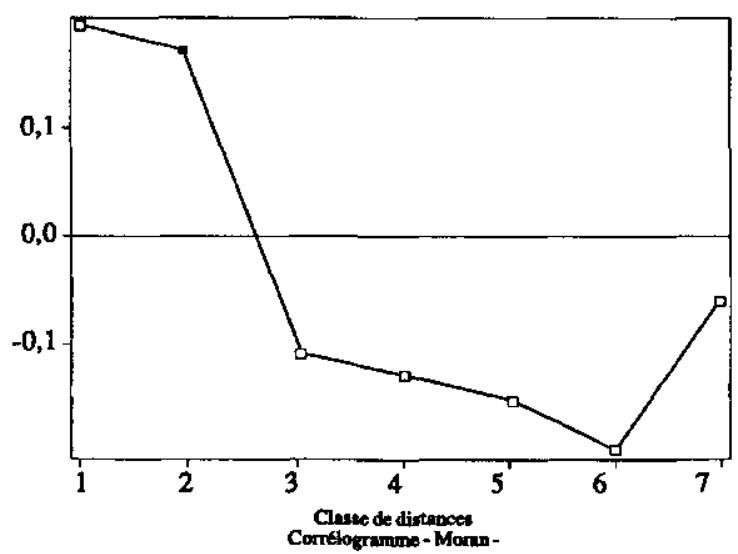

Figure 2 : Vols qualifiés, 11 mois 
significatives pour les classes de distance faibles et des valeurs négatives significatives pour les classes de distance fortes, et l'agglomerat (tache) ou la vague, caractérisée par un corrélogramme de Moran en «U»; les corrélogrammes de Geary ont une forme inversée. Legendre et Fortin (1989) ont simulé différents types de structure spatiale et discutent de l'interprétation des corrélogrammes.

La plupart des corrélogrammes que nous avons calculés étaient peu concluants. Pour les fichiers de données divisées en tranches de deux semaines, l'absence d'autocorrélation significative est certainement due au trop faible nombre d'observations contenues dans chaque période. Règle générale, l'absence de structure claire dans un corrélogramme spatial peut signifier qu'à l'échelle d'observation employée la surface étudiée est vraiment homogène et jouit donc de propriétés uniformes que l'on peut exploiter à des fins d'étude ou d'aménagement. En second lieu, elle peut également indiquer que la grille d'échantillonnage est inadéquate pour mettre en évidence la structure - gradient ou agrégation - éventuellement observable à une autre échelle. Enfin, puisque le corrélogramme décrit par une fonction unique la structure de toute la surface à l'étude, à cause d'une anisotropie ou du fait que plusieurs types de structure spatiale sont présents simultanément sur la surface à l'étude.

Le corrélogramme de Moran des données d'introduction par effraction cumulées sur 11 mois (figure 1) présente justement une structure mixte, qui ne peut être comprise que si on la compare à la carte (figure 6) qui sera discutée plus bas. La pente négative du corrélogramme correspond au gradient qui forme la structure spatiale est-ouest observable sur la carte; quant à la forme du corrélogramme en « $U$ " entre les classes de distance 1 et 5 , elle correspond vraisemblablement à la largeur de la structure en vague observable selon l'axe nord-sud. Il serait donc nécessaire de calculer des corrélogrammes indépendants dans ces deux directions pour identifier plus clairement ces structures spatiales.

Quant au corrélogramme de Moran calculé pour les données de vol qualifié cumulées sur 11 mois (figure 2), il montre principalement une forme en «U», qui correspondrait à la structure spatiale en vallée observable sur la carte de la figure 7 .

\section{b) Deuxième analyse: groupement sous contrainte}

Tel qu'il est expliqué à la section des «méthodes», nous avons opéré un regroupement des zones étudiées sous contrainte de contiguité 
spatiale (programme Biogéo), afin de vérifier la configuration la plus probable de la structure en termes de groupes homogenes; en d'autres termes, nous cherchons à mettre en évidence des plaques de densité analogue pour chacun des délits ainsi que pour la combinaison des deux délits. Ce traitement produit des résultats très nets.

Afin d'exprimer l'intensité d'occurrence des incidents selon les zones, nous présentons, aux tableaux I et II, les moyennes de zone calculées à partir du nombre d'événements dans les secteurs pour toute la période (11 mois). Il s'agit d'une simple préférence de présentation puisque après la procédure de regroupement les modes sont très évidents.

Tableau 1.

Regroupement des introductions par effraction

\begin{tabular}{|c|c|c|c|c|}
\hline Zone & A & B & C & D \\
\hline$\overline{\mathrm{x}}$ & 132,5 & 76,8 & 54,8 & 28,7 \\
\hline
\end{tabular}

Tableau 2.

Regroupement des vols qualifiés

\begin{tabular}{|c|c|c|c|c|}
\hline Zone & A & B & C & D \\
\hline$\overline{\overline{\mathrm{x}}}$ & 39,5 & 25,1 & 16,3 & $\mathbf{8 , 2 5 6}$ \\
\hline
\end{tabular}

Dans les deux cas, on observe des résultats très cohérents, conformes à ce que nous savons intuitivement du déroulement des événements dans ce district de police. La structure observée présente toutefois de brusques discontinuites; ce fait permet de mieux comprendre les résultats produits par les coefficients d'autocorrélation spatiale de Moran et de Geary. En effet, ces coefficients sont adaptés à l'analyse de l'autocorrélation dans un milieu isotrope et continu. Ces conditions ne sont pas présentes dans notre étude, comme en témoigne la cartographie des délits. Ainsi, l'autocorrélation se manifeste au sein des sections du territoire mais demeure plus difficilement mesurable pour l'ensemble, à cause de la résolution (c'est-à-dire, une zone) de l'échelle spatiale retenue pour cette étude.

La structure observée est stable lorsque l'on considère une période de 11 mois. En revanche, nous pouvons seulement présumer qu'elle s'est maintenue identique en très courte période. En effet, lorsque nous avons analysé l'évolution sur les surfaces par intervalle de deux se- 
maines, les trop faibles fréquences d'événements par zone n'ont pas permis de réaliser ce type d'analyse. Nous sommes ainsi amenés à considérer plusieurs hypothèses. La première serait que la méthode utilisée ne permet pas de capter le déplacement spatial de la criminalité. Nous devons écarter cette possibilité étant donné la qualité de la méthode de regroupement utilisée. Un autre point de vue admettrait que les déplacements dans cette zone se produiraient plus lentement que prévu, c'est-à-dire dans des intervalles de temps plus longs que ceux envisagés dans cette étude. Ceci est plausible mais ne peut se vérifier que par une réplique de cette étude dans la même zone sur une période beaucoup plus longue. Nous l'envisageons.

Comme autre hypothèse, on pourrait admettre l'existence d'une structure permanente ou quasi permanente qui exclurait toute turbulence dans le réel. Une telle proposition serait très osée, même si la permanence de la criminalité dans certains lieux est souvent évoquée et parfois observée. L'absence de turbulence demeure une possibilité qui compléterait notre problématique. Toutefois, nous nous abstiendrons de nous prononcer sur ce point avant d'avoir procédé aux observations que cette recherche désigne, c'est-à-dire l'évolution des moyennes dans les zones.

\section{c) Troisième analyse: surfaces théoriques}

L'analyse des surfaces théoriques conduit à des résultats particulièrement intéressants. Les coordonnées spatiales des centroïdes des 27 zones ont d'abord été centrées sur leur moyenne ( $x^{\prime}=x-2,926 ; y^{\prime}=$ y $-0,185$ ) avant le calcul des termes quadratiques et cubiques du polynôme (Équation 2).

Après la procédure d'élimination descendante, l'équation suivante a été trouvée pour les introductions par effraction (INTRO):

INTRO $=32,956-1,507\left(x^{\prime}\right)+3,234\left(y^{\prime}\right)-0,544\left(y^{\prime}\right)^{2}-0,058\left(y^{\prime}\right)^{3}$

Tous les coefficients de régression de cette équation sont significatifs ( $p \leq 0,01$ ). Cette équation spatiale explique $47,4 \%$ de la variation de la variable INTRO, ce qui représente une fraction hautement significative $(p=0,005)$.

Quant aux vols qualifiess (variable QUAL), la procédure d'élimination descendante conduit à l'équation suivante :

$\mathrm{QUAL}=59,136+6,530\left(\mathrm{y}^{\prime}\right)-0,061\left(\mathrm{x}^{\prime}\right)\left(\mathrm{y}^{\prime}\right)^{2}-0,059\left(\mathrm{y}^{\prime}\right)^{3}(4)$ 
Cette équation spatiale explique $31,2 \%$ de la variation de la variable QUAL, ce qui en représente une part significative $(p=0,032)$. Deux des trois coefficients de régression sont significatifs $\left[\left(y^{\prime}\right), p=0,016\right.$; $\left(x^{\prime}\right)\left(y^{\prime}\right)^{2}, p=0,026$. Quant au terme en $\left(y^{\prime}\right)^{3}$, quoique son coefficient de régression ( $p=0,081$ ) ne soit pas significatif au seuil de $5 \%$, il a quand même été conservé dans l'équation puisque son élimination faisait chuter le coefficient de détermination à $17,8 \%$ et le rendant non significatif.

Penchons-nous sur les résultats de cette modélisation spatiale pour les introductions par effraction; les valeurs prédites par le modele de régression sont cartographiées à la figure 3. Les cartes montrent d'abord l'existence d'un gradient linéaire d'est en ouest (correspondant au terme en (x') de l'équation (3) avec maximum à l'ouest; du nord au sud, la structure est plus complexe; elle croît depuis le secteur de la rue Sherbrooke pour atteindre son maximum sur la ligne reliant les stations de métro Place-des-Arts, Saint-Laurent, Berri-UQAM, Beaudry et Papineau, et s'abaisser de nouveau vers le fleuve ; cette structure correspond aux termes en $\left(y^{\prime}\right),\left(y^{\prime}\right)^{2}$ et $\left(y^{\prime}\right)^{3}$ de l'équation (3). Les valeurs maximales d'introduction par effraction prédites par ce modèle spatial se situent donc autour de la station de métro Place-des-Arts. Rappelons cependant que ce modèle n'explique que la moitié $(47,4 \%)$ de la variation des données; l'autre moitié, qui demeure pour le moment inexpliquée, pourait faire l'objet d'une modélisation plus détaillée à l'aide de variables indicatrices socio-économiques.

Les valeurs prédites par le modèle spatial de régression pour les vols qualifiés sont cartographieses à la figure 4 . Ce modèle, quoique statistiquement significatif, n'explique que le tiers environ de la variation de la variable à l'étude. La structure spatiale principale est une vague du nord au sud [correspondant aux termes $\left(y^{\prime}\right),\left(y^{\prime}\right)^{2}$ et $\left(y^{\prime}\right)^{3}$ de l'équation (4)], comme dans le modèle des introductions par effraction, auquel s'ajoute une remontée dans la zone 20 . De plus, la crête de la vague se situe au niveau des rues Sherbrooke (dans sa partie ouest, entre Jeanne-Mance et Laval) et Ontario (dans sa partie est), plutôt qu'autour du boulevard de Maisonneuve. Notons que le modèle prédit des valeurs minimales dans le corridor forme par la rue Saint-Catherine et le boulevard René-Lévesque ainsi que dans la partie sud-est du territoire à l'étude.

Nous devons mentionner que le contraste entre les résultats observés pour ces deux types de délits demeure difficile à interpréter. En effet, notre connaissance et nos appréciations des statistiques crimi- 
Nord

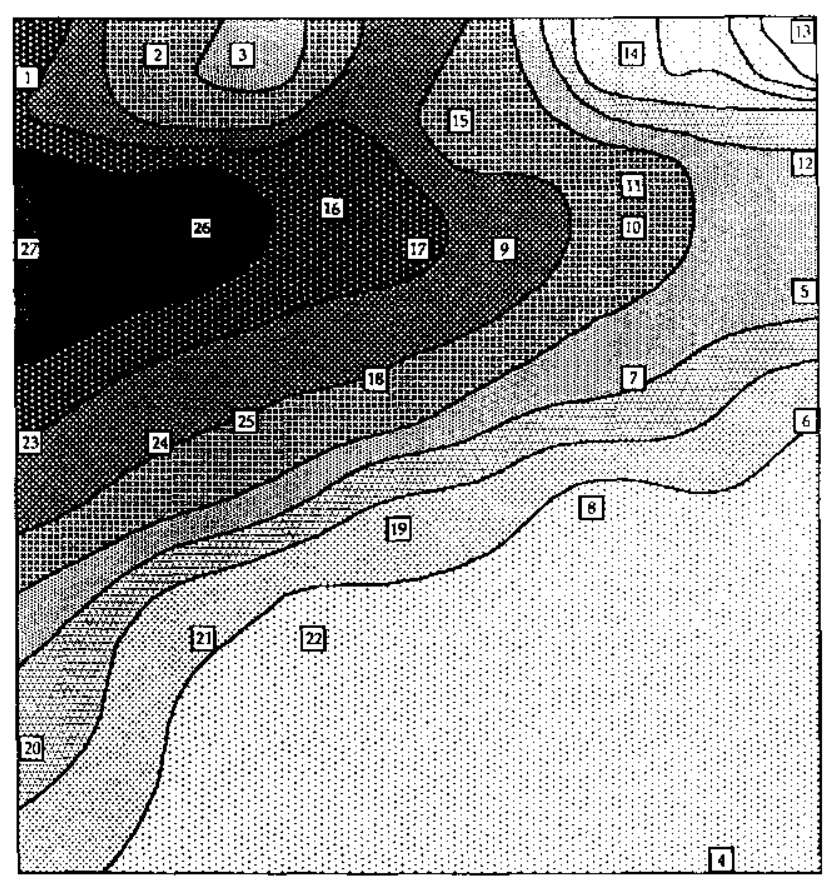

Sud

Introductions par effraction

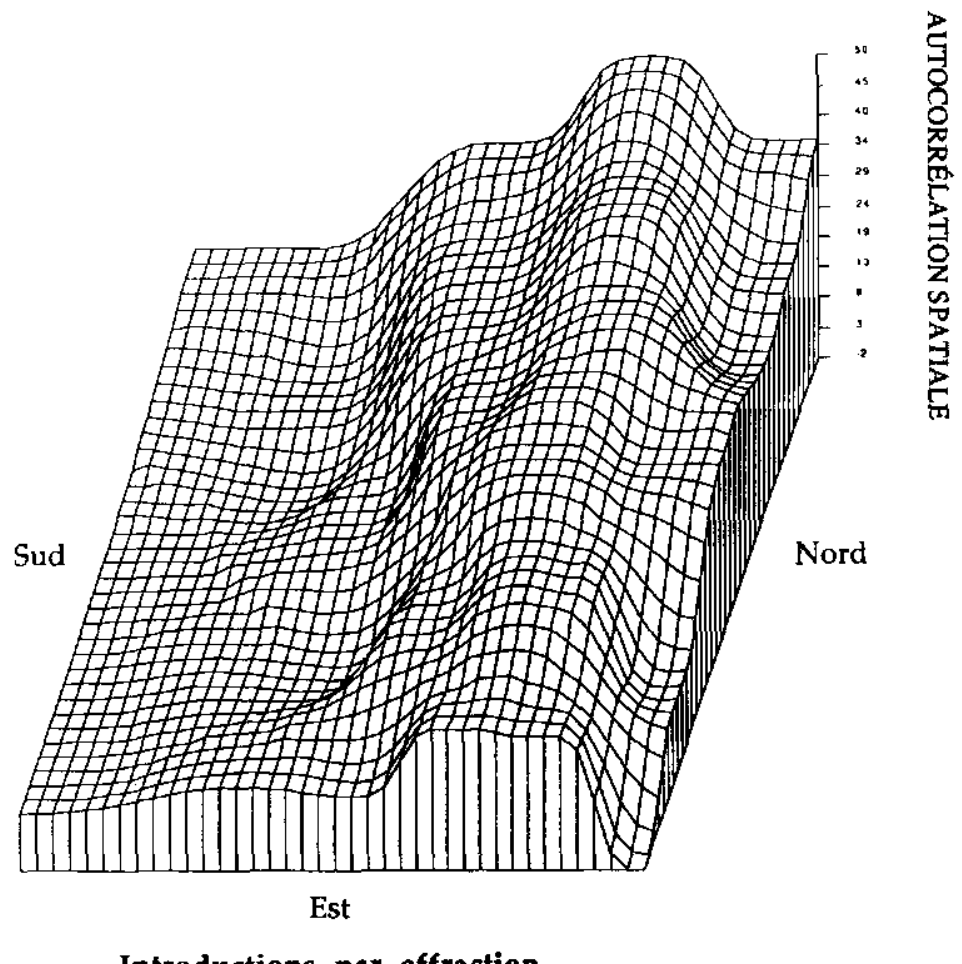

Est

Introductions par effraction

Figure 3 


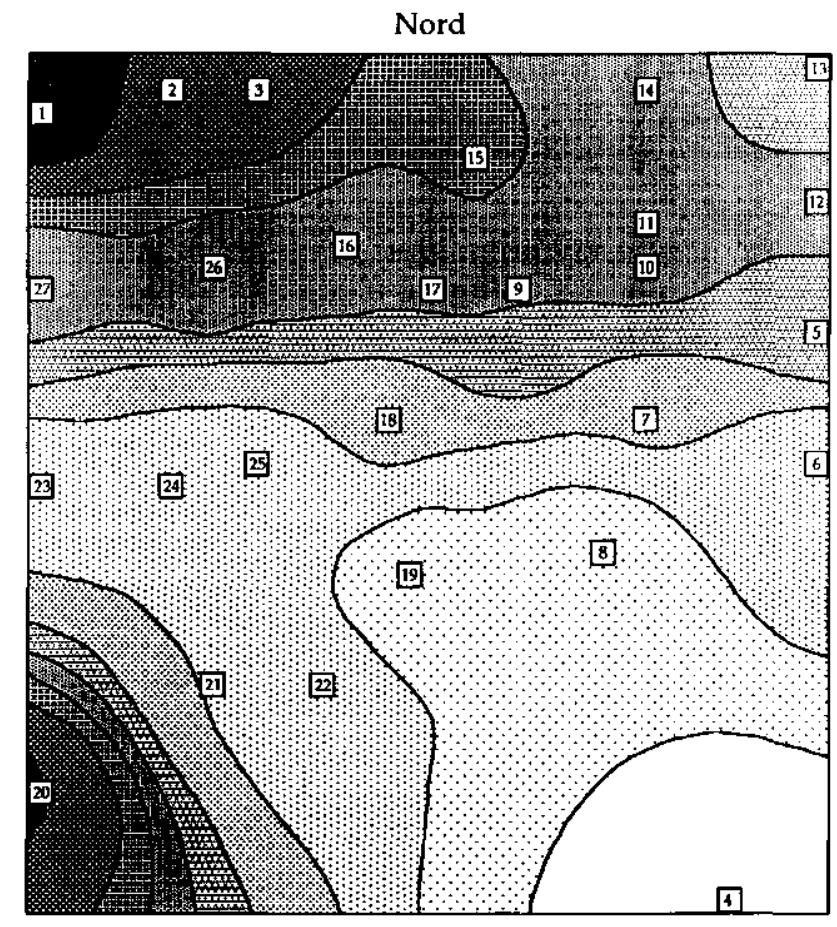

Sud

Vols qualifiés

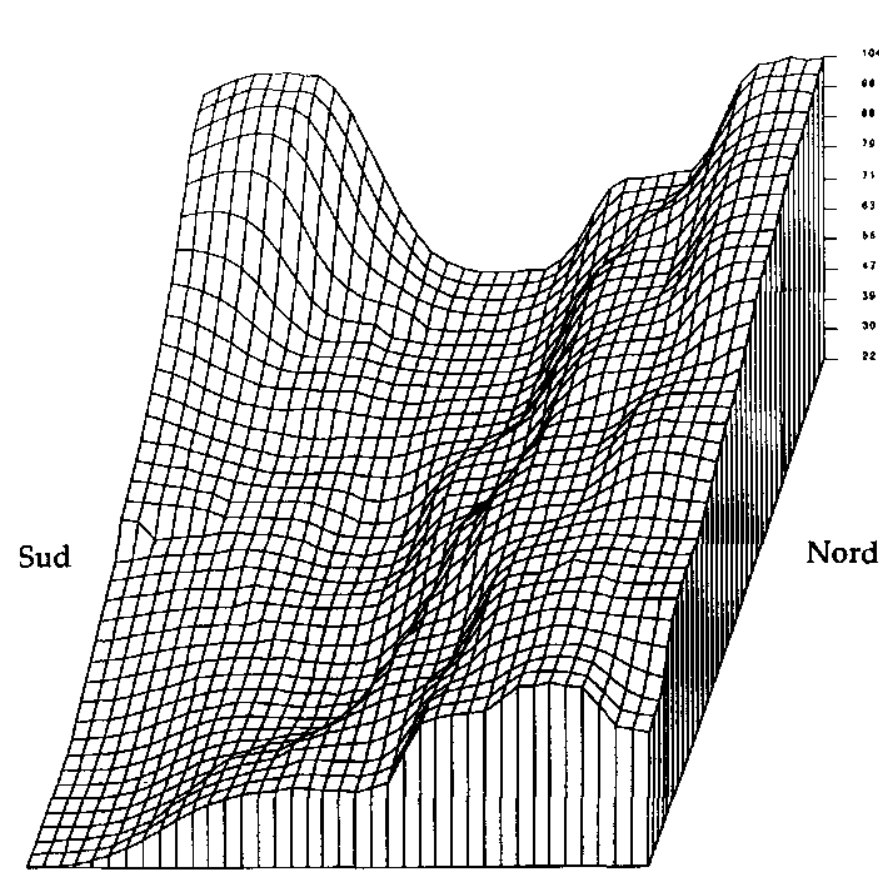

Est

Vols qualifiés 
nelles, notamment les taux différentiels de signalement de ces types de délits, nous invitent, dans cette première démarche, à une certaine prudence. En revanche, il sera très important et intéressant de combiner les deux dernières méthodes dans une réplique de cette étude en vue de l'observation du déplacement de la criminalité.

\section{CONCLUSION}

Les implications des faits que nous avons sont importants à plusieurs titres. Le premier point évident réside dans le constat de l'autocorrélation spatiale dans le secteur étudié. Il devient tout à fait raisonnable de penser qu'elle existe ailleurs avec ou sans périodicité ou turbulence. La question au sujet du déplacement demeure ouverte, mais nos résultats par rapport à l'autocorrélation représentent pour nous un acquis appréciable. Sur ce point, on peut constater que les trois méthodes utilisées diffèrent en termes de complexité et d'ađaptabilité aux données criminologiques. Il nous a semble que la cartographie illustrant le regroupement par contiguitté est d'une portée plus générale et répond le mieux à nos besoins en méthodologie actuellement.

Par ailleurs, il faut reconsidérer en conclusion un aspect important de notre problématique de départ. Il s'agit de ia praxis méthodologique usuelle dans laquelle on confronte les criminalités à des variables socioéconomiques et démographiques dans des analyses de concomitances ou de causalité. Toutes ou presque toutes ces variables sont liées à la spatialité. Or, nous constatons, à la fin de cette recherche, qu'au-delà des problèmes soulevés par les niveaux d'agrégation et les multicolinéarités il devient maintenant inévitable de considérer celui qu'implique la présence de l'autocorrélation spatiale. Nous rappelons qu'elle influence les épreuves de signification des statistiques le plus souvent dans le sens « libéral ». Finalement et à tout prendre, dans l'ignorance de ce fait, on a certainement interprété et on interprète encore erronément un nombre indiscernable de coefficients et de modèles. La logique et la prudence ordonnent de renoncer définitivement à ce type de recherche, à moins de tenir compte des faits évoqués dans ce texte.

La stratégie de recherche que nous préconisons est différente. Chaque fois qu'il est possible, procéder au type de regroupement spatial et de cartographie décrits ci-avant et utiliser le résultat dans une analyse comparative plus étendue des zones référées. L'utilisation des procédures de groupement et de cartographie peut aider à résoudre les problèmes posés par les niveaux d'agrégation spatiale. Le choix d'opérer 
par l'analyse et la comparaison de sous-zones homogènes devrait amener des résultats plus facilement interprétables.

Les suites à donner à la présente recherche nous semblent intéressantes. Il s'agira pour nous de trouver ce qui distingue les différentes zones avec des variables pertinentes au point de vue descriptif et theorique. Il sera également nécessaire de reprendre la même étude dans le même milieu au cours d'une autre période afin de pouvoir observer la stabilité de la structure ainsi que les types éventuels de déplacement.

\section{BIBLIOGRAPHIE}

BIVAND, R. (1980), A Monte Carlo study of correlation coefficient estimation with spatially auto-correlated observations, Quaest, Goergr., 6, p. 5-10.

BRANTINGHAM, P. J. et BRANTINGHAM, P. L. (1981), Environmental Criminology, Sage Publications.

CLIFF, A. D. et ORD, J. K. (1981), Spatial Processes: Models and Application, Pion Limited, London.

LEGENDRE, L. et LEGENDRE, P. (1984a), Écologie numérique, tome 2: La structure des données écologiques ( $2^{\bullet} e d$.), Masson, Paris et les Presses de l'Université du Québec.

LEGENDRE, P. (1987), «Constrained ciustering $"$, p. 289-307, in LEGENDRE L. et LEGENDRE P. (£d.), Developments in Numerial Ecology, NATO ASI Series, vol. G 14.

LEGENDRE, P. (1993), Spatial autocorrelation: Trouble or new paradigm?, Ecology (sous presse).

LEGENDRE, P. et FORTIN, M.-J. (1989), Spatial pattern and ecological analysis, Vegetatio, 80, p. 107-138.

LEGENDRE, P. et LEGENDRE, V. (1984b), Postglacial dispersal of freshwater fishes in the Québec peninsula, Can., J. Fish, Aquat. Sci., 41, p. 1781-1802.

RIPLEY, B. D. (1981), Spatial statistics. John Wiley \& Sons.

SHAW, C. R. et McKAY, H. D. (1931), Social Factors in Juvenile Delinquency, Washington, D.C., Government Printing Office. 